The Crossing Geometry of RHIC Insertion

S. Y. Lee

BNL

February 28, 1989 


\section{The Crossing Geometry of RHIC Insertion}

\section{S.Y. Lee}

The beam crossing geometry in RHIC insertion is tabulated and graphed here for reference. They may be useful in the particle tracking calculations and/or detector design. The beam is incident/or away from the triplet matching section with angle $3.97 \mathrm{mrad}$ at $23 \mathrm{~m}$ from the crossing point. The beam separation is determined to be $35 \mathrm{~cm}$ necessary for the dipole BC2 construction. Geometry is shown in Fig. 1. Since BC1 is a common dipole for both beams, unequal species colliding at the same velocity for synchronization would experience different bending kicks. The bending radius for these two species depends on their charge to mass ratio.

Defining the symbols $\alpha_{\mathrm{H}}, \mathrm{H} 1, \mathrm{H} 1^{\prime}, \mathrm{H} 12, \mathrm{H} 2$ shown in Fig. 1. The following table lists all relevant quantities with respect to $\alpha_{H}$. Some of these quantities are shown in Figs. 2 and 3. 


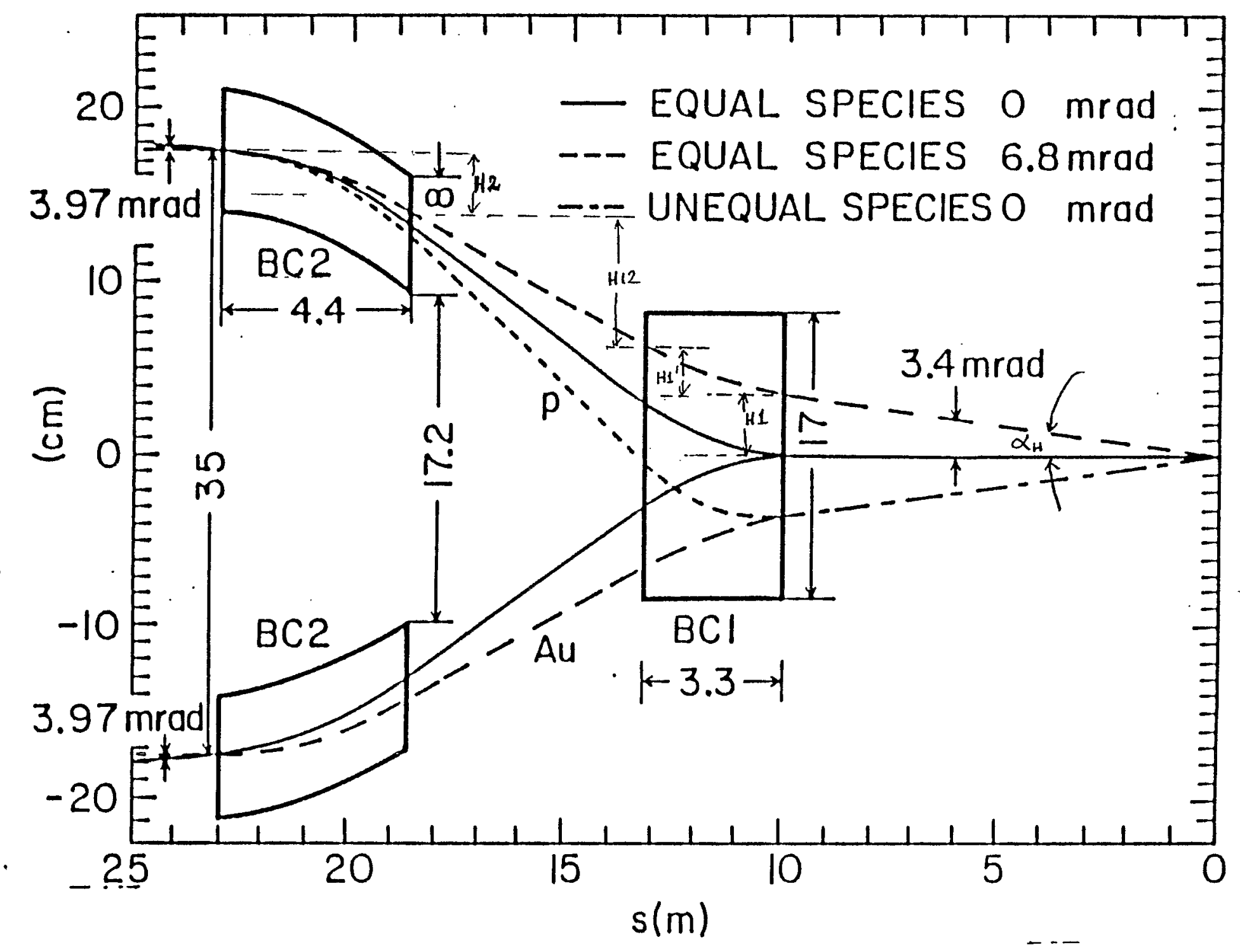




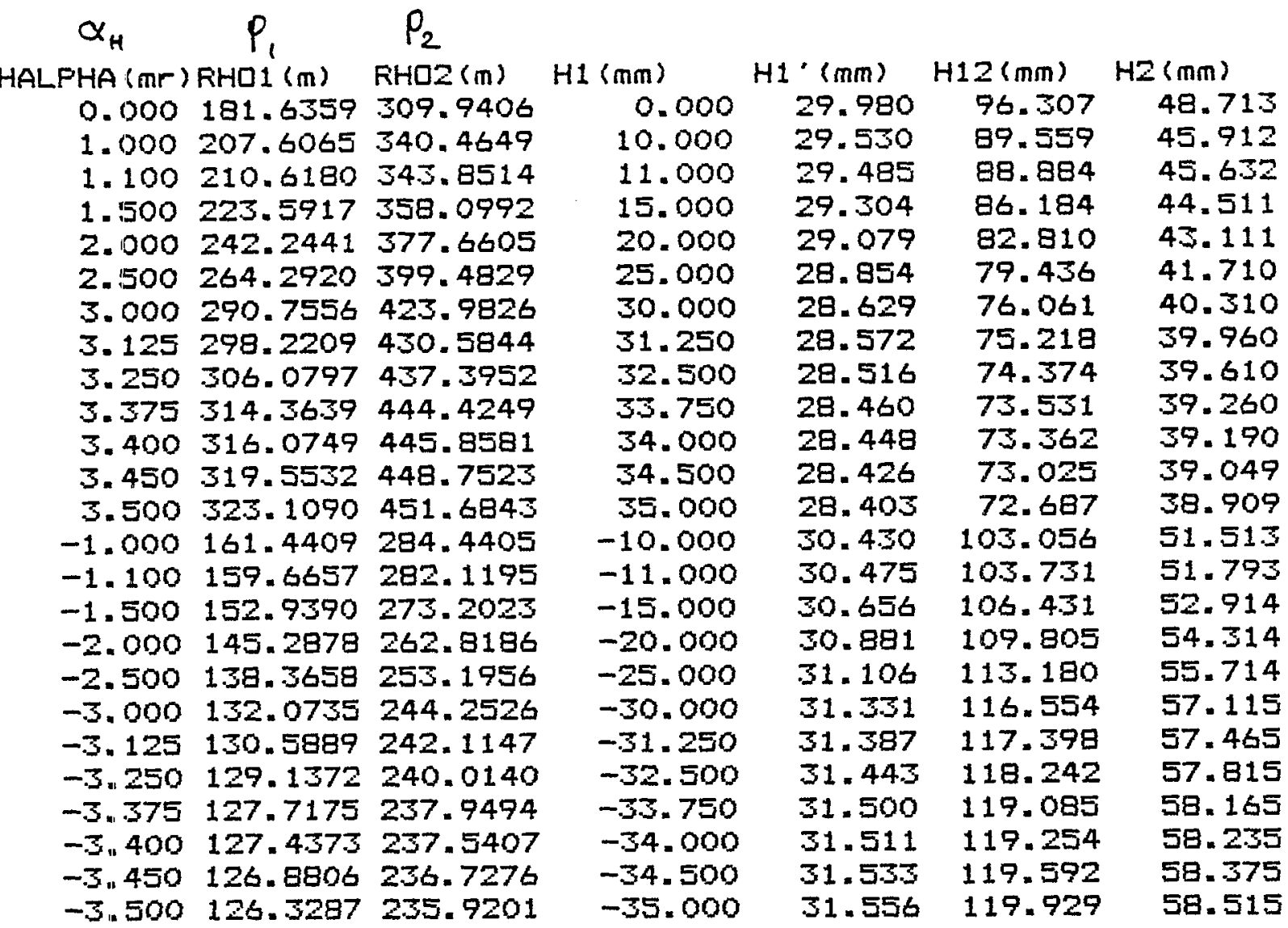

Beam sizes at various locations (mm) HEAVY ION

Emittance

GAMMA

BETA* (M)

PROTON

\begin{tabular}{|c|c|c|c|c|c|}
\hline $\begin{array}{l}34 \\
30\end{array}$ & & & $\begin{array}{r}34 \\
100\end{array}$ & & \\
\hline $\mathrm{BC} 12$ & $\mathrm{BC} 21$ & $\mathrm{BC2} 2$ & $\mathrm{BC} 12$ & $\mathrm{BC} 21$ & BC2Z \\
\hline 24.800 & 34.494 & 42.570 & 13.583 & 18.893 & 23.317 \\
\hline 20.527 & 28.365 & 34.921 & 11.243 & 15.536 & 19.127 \\
\hline $18=108$ & 24.806 & 30.438 & 9.918 & 13.587 & 16.672 \\
\hline 16.570 & 22.461 & 27.449 & 9.076 & 12.303 & 15.034 \\
\hline 15.533 & 20.806 & 25.305 & 8. 508 & 11.396 & 13.86 \\
\hline
\end{tabular}

\begin{tabular}{|c|c|c|c|c|c|c|}
\hline $\begin{array}{l}\text { Emittance } \\
\text { GAMMA }\end{array}$ & $\begin{array}{l}16 \\
30\end{array}$ & & & $\begin{array}{r}17 \\
100\end{array}$ & & \\
\hline BETA* (M) & $\mathrm{BC} 12$ & $B C 21$ & $\mathrm{BC} 22$ & $\mathrm{BC} 12$ & $\mathrm{BC} 21$ & $\mathrm{BC} 22$ \\
\hline $\begin{array}{l}2 \\
3 \\
4 \\
5 \\
6\end{array}$ & $\begin{array}{l}17.012 \\
14.081 \\
12.422 \\
11.367 \\
10.656\end{array}$ & $\begin{array}{l}23.663 \\
19.458 \\
17.017 \\
15.408 \\
14.273\end{array}$ & $\begin{array}{l}29.203 \\
23.956 \\
20.881 \\
18.830 \\
17.359\end{array}$ & $\begin{array}{l}9.605 \\
7.950 \\
7.013 \\
6.418 \\
6.016\end{array}$ & $\begin{array}{r}13.360 \\
10.986 \\
7.607 \\
8.699 \\
8.058\end{array}$ & $\begin{array}{r}16.487 \\
13.525 \\
11.789 \\
10.631 \\
9.801\end{array}$ \\
\hline
\end{tabular}

$B C 12:$ End of $B C 1$

$B C 21$ : BEGINING of $B C 2$

8C22: END of $B C 2$ 


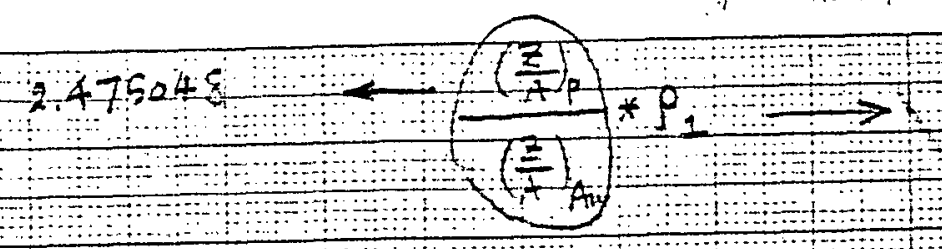$$
E \text { 河 } B P=04 \%
$$

\section{Trala}

$p_{1}(m)$

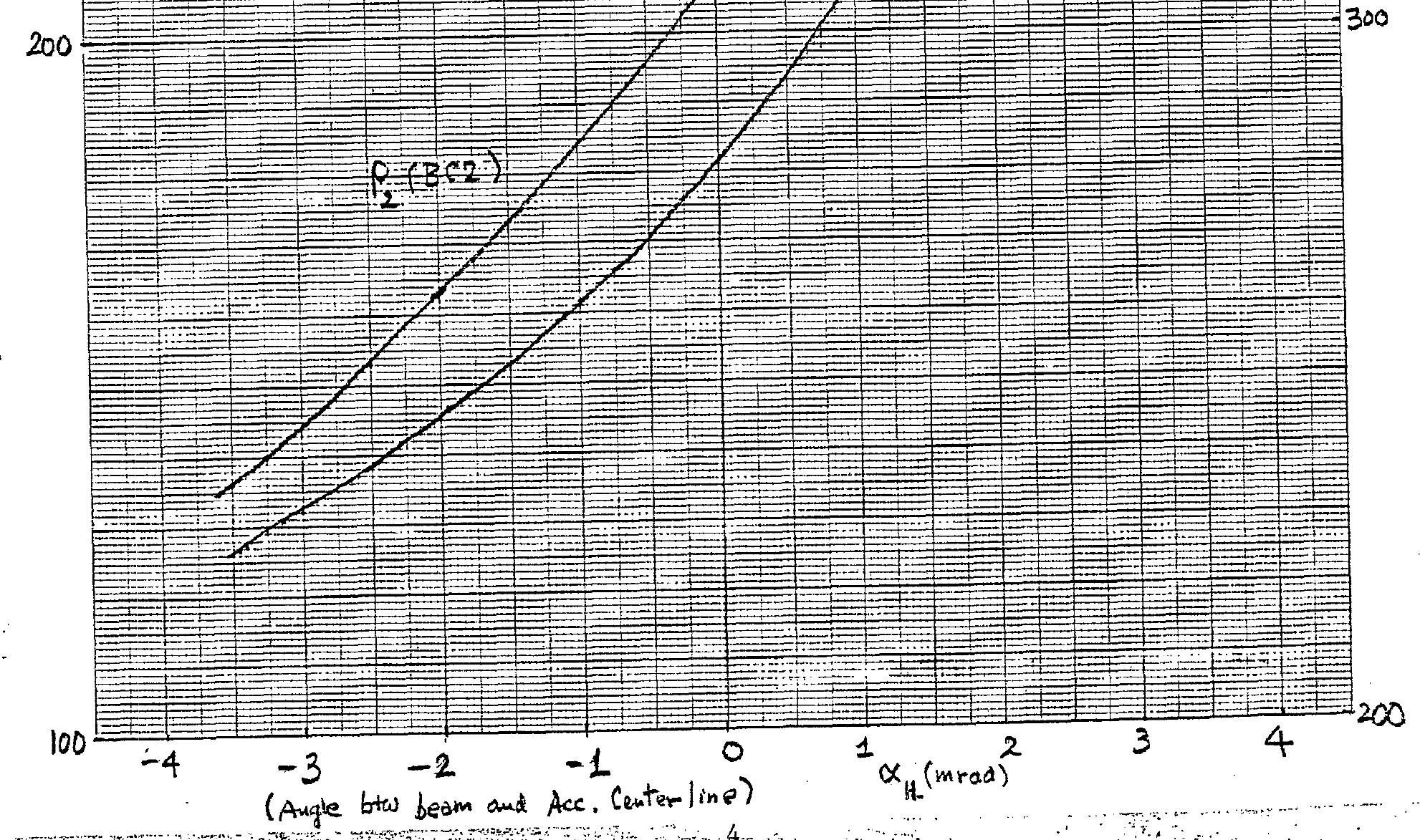


$\frac{0}{5}$

mm

$4+4$

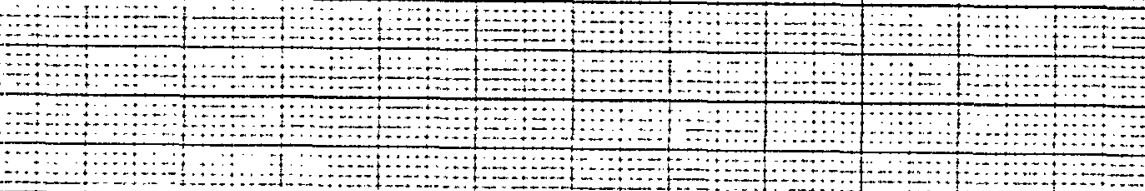

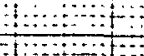

$1+$

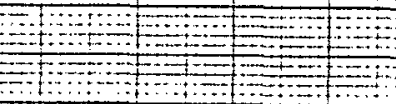

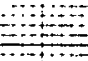

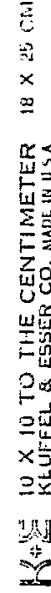

150

$+3=5=5$

$=0$

$\frac{1}{0}$

0

$\frac{2}{2}$

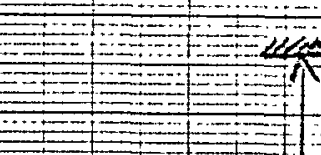

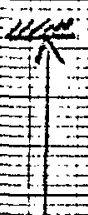

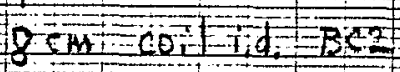

100

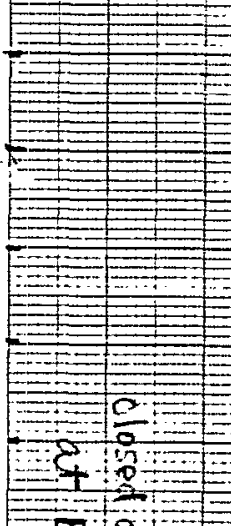

50

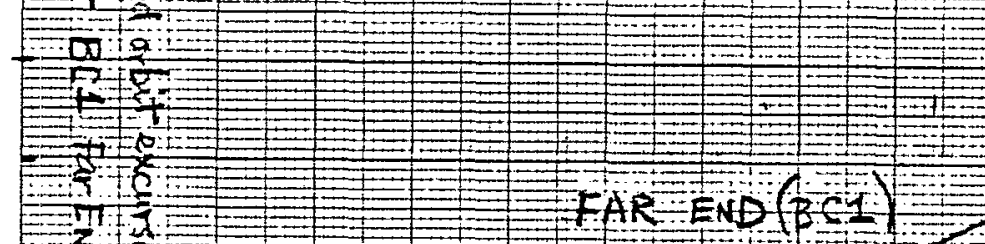

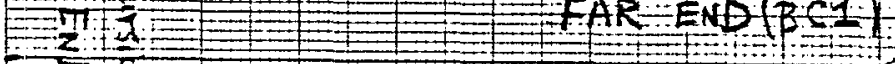

$=0.5$

NEAR LSG of $B C Z$

$1=$

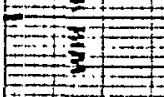

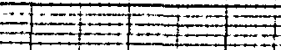

$\sqrt{27 \pi}$
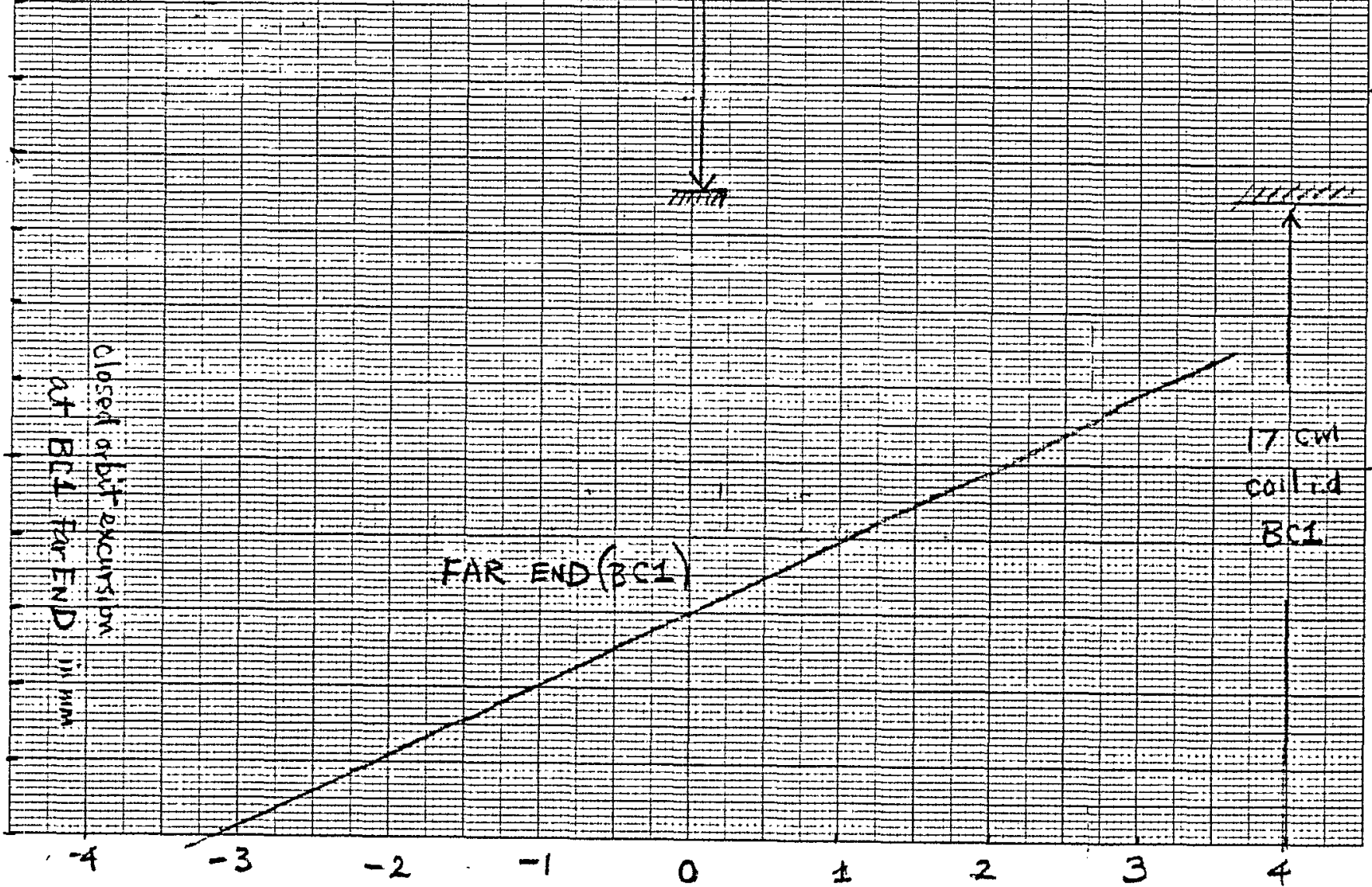

$-4$

$-3$

$-2$

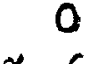

0

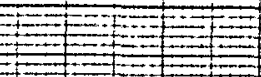

12

$x+1=$

$\alpha_{H}(\mathrm{mrad})$ 